\title{
Clostridium botulinum type A haemagglutinin- positive progenitor toxin (HA+-PTX) binds to oligosaccharides containing Gal/31-4GIcNAC through one subcomponent of haemagglutinin (HA1)
}

\author{
Kaoru Inoue, ${ }^{1}$ Yukako Fujinaga, ${ }^{1}$ Koichi Honke, ${ }^{2}$ Hideyuki Arimitsu, ${ }^{1}$ \\ Nazira Mahmut, ${ }^{1}$ Yoshihiko Sakaguchi, ${ }^{1}$ Tohru Ohyama, ${ }^{3}$ \\ Toshihiro Watanabe, ${ }^{3}$ Katsuhiro Inoue $^{3}$ and Keiji Oguma ${ }^{1}$
}

1 Department of Bacteriology, Okayama University Medical School, 2-5-1 Shikata-cho, Okayama 700-8558, Japan

2 Department of Biochemistry, Osaka University Medical School, 2-2 Yamadaoka, Suita, Osaka 565-0871, Japan

3 Department of Food Science, Faculty of Bioindustry, Tokyo University of Agriculture, 196 Yasaka, Abashiri 099-2422, Japan
Author for correspondence: Keiji Oguma. Tel: +8186235 7162. Fax: +81 862357162. e-mail:kuma@med.okayama-u.ac.jp

Haemagglutinin (HA) activity of Clostridium botulinum type A 195 and 165 toxins (HA-positive progenitor toxin; HA+-PTX) was characterized. HA titres against human erythrocytes of HA+-PTX were inhibited by the addition of lactose, D-galactose, $\boldsymbol{N}$-acetyl-D-galactosamine and D-fucose to the reaction mixtures. A direct glycolipid binding test demonstrated that type A HA+-PTX strongly bound to paragloboside and some neutral glycolipids, but did not bind to gangliosides. Type A HA+-PTX also bound to asialoglycoproteins (asialofetuin, neuraminidase-treated transferrin), but not to sialoglycoproteins (fetuin, transferrin). Although glycopeptidase $F$ treatment of asialofetuin abolished the binding of HA+-PTX, endo- $\alpha$ - $N$-acetylgalactosaminidase treatment did not. Thus these results can be interpreted as indicating that type A HA+-PTX detects and binds to Gal/11-4GIcNAc in paragloboside and the $\boldsymbol{N}$-linked oligosaccharides of glycoproteins. Regardless of neuraminidase treatment, type A HA+-PTX bound to glycophorin A which is a major sialoglycoprotein on the surface of erythrocytes. Both native glycophorin $A$ and neuraminidasetreated glycophorin A inhibited the binding of erythrocytes to type A HA ${ }^{+}$-PTX. Since the $\boldsymbol{N}$-linked oligosaccharide of glycophorin $\mathrm{A}$ is di-branched and more than $50 \%$ of this sugar chain is monosialylated, type A HA ${ }^{+}$-PTX probably bound to the unsialylated branch of the $\boldsymbol{N}$-linked oligosaccharide of glycophorin A and agglutinated erythrocytes. One subcomponent of HA, designated HA1, did not agglutinate native erythrocytes, although it did bind to erythrocytes, paragloboside and asialoglycoproteins in a manner quite

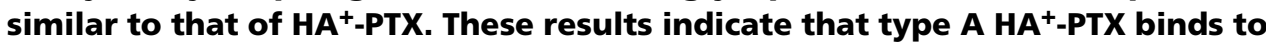
oligosaccharides through HA1.

Keywords: binding, sugar chain, glycolipid, glycoprotein

\section{INTRODUCTION}

Clostridium botulinum strains produce immunologically distinct neurotoxins (type A to G) which inhibit the release of acetylcholine at neuromuscular junctions and synapses. The molecular masses of all types of these neurotoxins are approximately $150 \mathrm{kDa}$. The neuro-

Abbreviations: $\mathrm{CBB}$, Coomassie brilliant blue; $\mathrm{HA}$, haemagglutinin; $\mathrm{HA}^{+-}$ PTX, haemagglutinin-positive progenitor toxin; PAS, periodic acid-Schiff. toxins associate with non-toxic components in culture fluids to form larger complexes which are designated progenitor toxins. In the type A strain, three different sized progenitor toxins with molecular masses of $900 \mathrm{kDa}(19 \mathrm{~S}), 500 \mathrm{kDa}(16 \mathrm{~S})$ and $300 \mathrm{kDa}(12 \mathrm{~S})$ are produced. Type B, C and D strains produce both the $16 \mathrm{~S}$ and $12 S$ toxins, whereas the type $\mathrm{E}$ and $\mathrm{F}$ strains produce only the $12 \mathrm{~S}$ toxin and type $\mathrm{G}$ strain produces only the $16 S$ toxin (Sakaguchi et al., 1984). In all these strains, the non-toxic components of the 195 and 16 S toxins display 
haemagglutinin (HA) activity but that of the $12 \mathrm{~S}$ toxin does not. The $12 \mathrm{~S}$ toxin is formed by association of a neurotoxin with a non-toxic component having no HA activity (designated here as non-toxic non-HA), while $19 \mathrm{~S}$ and $16 \mathrm{~S}$ toxins are formed by conjugation of the $12 \mathrm{~S}$ toxin with HA. The non-toxic components (HA and non-toxic non-HA) are considered to be very important to the development of food poisoning because the nontoxic components protect the neurotoxin from the barrier of gastric juice when the progenitor toxins pass through the stomach (Sugii et al., 1977). Therefore, the larger the molecular size of the progenitor toxin of the same type, the higher the oral toxicity (Ohishi \& Sakaguchi, 1980; Ohishi et al., 1977).

We purified different sized progenitor toxins from Clostridium botulinum type A and C culture fluids, and demonstrated that the HAs consist of subcomponents that have molecular masses of 52-53, 33-35, 19-23 and 15-17 kDa, which are designated here HA3b, HA1, HA3a and HA2, respectively (Fujinaga et al., 1994; Inoue et al., 1996). In a previous study, we purified types $C$ and D 16S toxins, and characterized their HA activity and binding to glycolipids and glycoproteins (Inoue et al., 1999). Types C and D $16 S$ toxins agglutinated human erythrocytes and both HA activities were reduced by employing erythrocytes that had been treated with neuraminidase. Types C and D $16 \mathrm{~S}$ toxins bound to sialylglycolipids and sialoglycoproteins but did not bind to neutral glycolipids or asialoglycoproteins. In the present study, we performed haemagglutination and haemagglutination-inhibition tests, and direct binding tests to glycolipids and glycoproteins, by employing purified type A HA+-PTX and HA1 to determine how they bind to erythrocytes.

\section{METHODS}

Bacteria and toxin. C. botulinum type A strain A-NIH was used. The mixture of type A 195 and 16 S toxins, designated here as type $\mathrm{A} \mathrm{HA}^{+}$-PTX, was purified from culture fluid (Inoue et al., 1996). Partially purified type A HA1 was further purified with a hydroxyapatite (Seikagaku) column $(0.7 \times 5 \mathrm{~cm})$ equilibrated with $10 \mathrm{mM}$ phosphate buffer $(\mathrm{pH} 6.0)$ and eluted with $10 \mathrm{mM}$ phosphate buffer containing $1 \mathrm{M} \mathrm{NaCl}$ (Fig. 1). We previously reported that C. botulinum type A culture fluid contained $\mathrm{HA}^{+}-\mathrm{PTX}$ (19S and $16 \mathrm{~S}$ toxins), $\mathrm{HA}^{-}$-PTX (12S toxin), free HA and free HA1 (Inoue et al., 1996). The HA or $\mathrm{HA}^{+}-\mathrm{PTX}$ contained in the HA1 sample was eluted with $0 \cdot 2 \mathrm{M}$ phosphate buffer $(\mathrm{pH} 6 \cdot 0)$ and possessed HA activity.

Glycolipids and glycoproteins. Glycosphingolipids were purified from the following sources as described previously by Gasa et al. (1983): GM4 (NeuAc $\alpha 2-3 \mathrm{Gal} \beta 1-\mathrm{Cer}$ ), GM1 [Gal $\beta 1-3 \mathrm{GalNAc} \beta 1-4(\mathrm{NeuAc} \alpha 2-3) \mathrm{Gal} \beta 1-4 \mathrm{Glc} \beta 1-\mathrm{Cer}]$, GD1a [NeuAc $\alpha 2-3$ Gal $\beta 1-3$ GalNAc $\beta 1-4$ (NeuAc $\alpha 2-3)$ Gal $\beta 1-$ 4Glc $\beta 1$-Cer], GD1b [Gal $\beta 1-3 \mathrm{GalNAc} \beta 1-4$ (NeuAc $\alpha 28 \mathrm{Neu}-$ Ac $\alpha 2-3) \mathrm{Gal} \beta 1-4 \mathrm{Glc} \beta 1$-Cer $]$ and SM4 (HSO $-3 \mathrm{Gal} \beta 1-\mathrm{Cer})$ from bovine brain; GM2 [GalNAc $\beta 1-4(\mathrm{NeuAc} \alpha 2-3) \mathrm{Gal} \beta 1$ 4Glc $\beta 1$-Cer] from a Tay-Sachs brain; ceramide monohexoside (a mixture of Gal $\beta 1$-Cer and Glc $\beta 1$-Cer) and LacCer (Gal $\beta 1-4 \mathrm{Glc} \beta 1$-Cer) from horse erythrocytes; Gb3Cer (Gal $\alpha 1-4 \mathrm{Gal}_{\beta} 1-4 \mathrm{Glc} \beta 1-\mathrm{Cer}$ ) and SM3 (HSO ${ }_{3}-3 \mathrm{Gal} \beta 1-4 \mathrm{Glc} \beta 1$ Cer) from human kidney; Gb4Cer (GalNAc $\beta 1-3 \mathrm{Gal} \alpha 1$ -

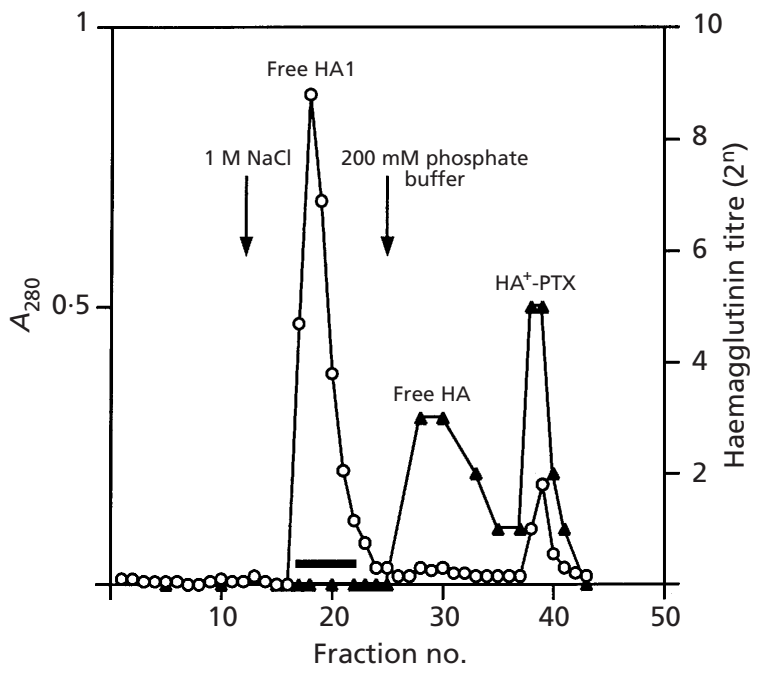

Fig. 1. Hydroxyapatite column chromatography. The sample was applied to a hydroxyapatite column equilibrated with $10 \mathrm{mM}$ phosphate buffer (pH 6.0). After washing the column with 1.5 column vols equilibrating buffer, the elution was performed successively with $10 \mathrm{mM}$ phosphate buffer (pH 6.0) containing $1 \mathrm{M} \mathrm{NaCl}$ and $200 \mathrm{mM}$ phosphate buffer $(\mathrm{pH} \mathrm{6.0)}$ ). Fractions of $1 \mathrm{ml}$ were collected. The fractions indicated by a solid bar were pooled. $O, A_{280} ; \boldsymbol{\Delta}, \mathrm{HA}$ titre.

$4 \mathrm{Gal} \beta 1-4 \mathrm{Glc} \beta 1$-Cer) and SPG (NeuAc $\alpha 2-3 \mathrm{Gal} \beta 1-4 \mathrm{GlcNac} \beta$ $3 \mathrm{Gal} \beta 1-4 \mathrm{Glc} \beta 1-\mathrm{Cer})$ from human erythrocytes; and GM3 (NeuAc $\alpha 2-3 \mathrm{Gal} \beta 1-4 \mathrm{Glc} \beta 1-\mathrm{Cer})$ and GD3 (NeuAc $\alpha 2-$ $8 \mathrm{NeuAc} \alpha 2-3 \mathrm{Gal} \beta 1-4 \mathrm{Glc} \beta 1$-Cer) from rat liver. Asialo GM1 (bovine brain) was purchased from Wako Pure Chemical Industries. Paragloboside was obtained by treatment of SPG with neuraminidase. Fetuin was purchased from Wako Pure Chemical Industries. Asialofetuin and glycophorin (from blood type $\mathrm{MN}$, predominantly glycophorin A) were purchased from Sigma.

SDS-PAGE and electroblotting. SDS-PAGE was performed by the method of Laemmli (1970) using $12.5 \%$ acrylamide linear gels. Protein bands were stained with Coomassie brilliant blue (CBB) R-250. The molecular mass markers used were myosin $(200 \mathrm{kDa}), \beta$-galactosidase $(116 \mathrm{kDa})$, phosphorylase $b$ $(97 \cdot 4 \mathrm{kDa})$, BSA $(66 \cdot 2 \mathrm{kDa})$, ovalbumin $(45 \mathrm{kDa})$, carbonic anhydrase $(31 \mathrm{kDa})$, soybean trypsin inhibitor $(21.5 \mathrm{kDa})$ and lysozyme $(14.4 \mathrm{kDa})$. The bands separated by SDS-PAGE were electroblotted to PVDF membranes (Immobilon; Millipore) with a semidry blotting apparatus (Nippon Eido) according to the methods described by Hirano \& Watanabe (1990).

Preparation of antisera and immunoblotting. Antiserum against type A HA prepared previously in rabbit was employed (Inoue et al., 1996). Antiserum against type A HA1 was also prepared in rabbit by the method described previously (Inoue et al., 1996). Immunoblotting was performed as described previously (Inoue et al., 1999).

Enzyme treatment of human erythrocytes. Ten millilitre aliquots of $10 \%(\mathrm{v} / \mathrm{v})$ washed human erythrocytes were incubated with $0 \cdot 1 \mathrm{U}$ Arthrobacter ureafaciens neuraminidase (Nacalai tesque), at $37^{\circ} \mathrm{C}$ for $1 \mathrm{~h}$. After treatment, the erythrocytes were washed three times in PBS (pH 6.0). 
Determination of HA titre and inhibition tests. The HA titre was obtained by microtitration methods using multiwell plates as described previously (Inoue et al., 1999). Inhibition of HA activity of the type A $\mathrm{HA}^{+}$-PTX with several different saccharides was examined as follows. Each diluted preparation $(20 \mu \mathrm{l})$ was mixed with $20 \mu \mathrm{l}$ of an appropriate concentration of saccharide solution and incubated at $37{ }^{\circ} \mathrm{C}$ for $1 \mathrm{~h}$. Thereafter, $40 \mu \mathrm{l} 1 \%$ (v/v) erythrocyte suspension was added to the mixtures. Following incubation at room temperature for $2 \mathrm{~h}$, haemagglutination was assessed. All tests were performed in duplicate and repeated twice.

TLC immunostaining. Binding of type $\mathrm{A} \mathrm{HA}^{+}$-PTX and HA1 to the glycolipids was analysed by TLC immunostaining as described previously (Inoue et al., 1999) with some modifications. The glycolipids were developed on high-performance thin-layer chromatography (HPTLC) aluminium sheet silica gel 60 plates (Merck) with chloroform/methanol/water $(65: 35: 8$, by vol.). The developed plates were dipped in $0.2 \%$ $(\mathrm{w} / \mathrm{v})$ polyisobutyl-methacrylate in $\mathrm{n}$-hexane for $1 \mathrm{~min}$ and dried. The plates were incubated in PBS ( $\mathrm{pH} \mathrm{6.0)} \mathrm{containing}$ $3 \%(\mathrm{w} / \mathrm{v})$ BSA (blocking buffer 1 ) for $1 \mathrm{~h}$, followed by incubation with $10 \mu \mathrm{g}$ type $\mathrm{A} \mathrm{HA}^{+}-\mathrm{PTX} \mathrm{ml} \mathrm{H}^{-1}$ or $100 \mu \mathrm{g}$ HA1 $\mathrm{ml}^{-1}$ (in blocking buffer 1 ) for $1 \mathrm{~h}$. The plates were then washed three times with PBS $(\mathrm{pH} 6.0)$ containing $0.05 \%(\mathrm{v} / \mathrm{v})$ Tween 20 (PBS/Tween) and reacted for $1 \mathrm{~h}$ with antiserum against type A HA or antiserum against type A HA1 diluted $1: 1000$ with blocking buffer 1 . After washing three times with PBS/Tween, the plates were incubated with a peroxidaselabelled anti-rabbit IgG antibody (DAKO A/S) diluted 1:1000 with blocking buffer 1 for $1 \mathrm{~h}$. The immunoreactive bands were detected by the enhanced chemiluminescence Western blotting (immunoblotting) system (ECL; Amersham). All of the procedures were performed at room temperature.

Enzyme treatment of glycoproteins. Enzyme treatment of glycoproteins was performed as follows. Transferrin $(25 \mu \mathrm{g})$ or glycophorin $(25 \mu \mathrm{g})$ was incubated with neuraminidase $(2.5 \mathrm{mU})$ in the reaction mixture $(25 \mu \mathrm{l})$ containing $10 \mathrm{mM}$ phosphate buffer $(\mathrm{pH} 7 \cdot 4)$ and $0.15 \mathrm{M} \mathrm{NaCl}$ at $37^{\circ} \mathrm{C}$ overnight. Transferrin and asialofetuin $(25 \mu \mathrm{g}$ each) were incubated with glycopeptidase F ( $1 \mathrm{mU}$, Takara Shuzo) in the reaction mixture $(25 \mu \mathrm{l})$ according to the instruction manual at $37^{\circ} \mathrm{C}$ overnight. Asialofetuin $(25 \mu \mathrm{g})$ was incubated with endo- $\alpha-N$-acetylgalactosaminidase $(2.5 \mathrm{mU}$, Seikagaku $)$ in a reaction mixture containing $20 \mathrm{mM}$ citrate buffer $(\mathrm{pH} 4.5)$ at $37^{\circ} \mathrm{C}$ for $20 \mathrm{~min}$.

Binding to ghost membrane proteins or glycoproteins. We analysed the binding of type A HA ${ }^{+}$-PTX to ghost membrane proteins and glycoproteins. The PVDF membranes blotted with proteins were immersed overnight in PBS (pH 6.0) containing $10 \%(\mathrm{w} / \mathrm{v})$ BSA (blocking buffer 2 ) at $4{ }^{\circ} \mathrm{C}$. The membranes were incubated in $10 \mu \mathrm{g}$ type $\mathrm{A} \mathrm{HA}^{+}-\mathrm{PTX} \mathrm{ml}{ }^{-1}$ or $100 \mu \mathrm{g} \mathrm{HA} 1 \mathrm{ml}^{-1}$ in blocking buffer 2 for $2 \mathrm{~h}$ and then washed three times with PBS/Tween. Bound type $\mathrm{A} \mathrm{HA}^{+}$-PTX and HA1 to glycoproteins were detected by antiserum against type A HA and antiserum against type A HA1, respectively, and the immunoreactive bands were detected by ECL (Amersham).

Binding of erythrocytes to toxins. The binding of type $\mathrm{A} \mathrm{HA}^{+}-$ PTX and HA1 to erythrocytes was analysed using 96-well microtitration plates as previously described by Hoschutzky et al. (1989) with minor modification. Fifty microlitre aliquots of $\mathrm{HA}^{+}$-PTX or HA1 $\left(10 \mu \mathrm{g} \mathrm{ml}^{-1}\right)$ were incubated in microtitre plates and left to stand overnight at $4^{\circ} \mathrm{C}$. The plates were washed three times with $200 \mu \mathrm{l}$ PBS (pH 6.0), then $100 \mu \mathrm{l}$ PBS ( $\mathrm{pH} 6.0)$ containing $1 \%$ BSA was added to each well and incubated for $2 \mathrm{~h}$ at room temperature. After washing the wells three times with $200 \mu \mathrm{l}$ PBS (pH 6.0), $100 \mu \mathrm{l}$ PBS (pH 6.0) containing glycoprotein was added to each well. After $1 \mathrm{~h}$ incubation at room temperature, $10 \mu 110 \%(\mathrm{v} / \mathrm{v})$ native or neuraminidase-treated erythrocytes in PBS (pH 6.0) was added. Plates were incubated for $30 \mathrm{~min}$ at room temperature and washed six or seven times with $200 \mu \mathrm{l} \mathrm{PBS} \mathrm{(pH} \mathrm{6.0).} \mathrm{Bound}$ erythrocytes were lysed by adding $50 \mu \mathrm{l}$ distilled water and the absorbance at $405 \mathrm{~nm}$ was analysed. All tests were performed in duplicate and repeated three times.

\section{RESULTS AND DISCUSSION}

\section{SDS-PAGE and immunoblot analysis of $\mathrm{HA}^{+}-$PTX and HA1}

Type A HA ${ }^{+}$-PTX and HA1 were subjected to SDSPAGE. HA ${ }^{+}$-PTX showed seven major bands (Fig. 2a, lane 4). On the basis of our previous report, each protein band was assigned as non-toxic non-HA, heavy chain of neurotoxin, HA3b, light chain of neurotoxin, HA1, HA3a and HA2, respectively (Inoue et al., 1996). Type A HA1 demonstrated a single band with a molecular mass of $35 \mathrm{kDa}$ (Fig. 2a, lane 3). The mobility of this protein band was the same as that of HA1 of $\mathrm{HA}^{+}-\mathrm{PTX}$. When HA1 was subjected to SDS-PAGE without heat treatment, a single protein band with molecular mass of $60 \mathrm{kDa}$ was evident by CBB staining (Fig. 2a, lane 2). The antiserum against type A HA reacted with $\mathrm{HA} 3 \mathrm{~b}$, HA1 and HA3a of $\mathrm{HA}^{+}$-PTX, and free HA1 as reported previously (Fig. 2c, lanes 3 and 4; Inoue et al., 1996). In the preparation of non-heated free HA1, two bands of 60 and $30 \mathrm{kDa}$ appeared with either antiserum against type A HA or HA1. Thus, free HA1 may exist as a dimer in culture fluid and the native HA1 monomer may have more a compact form than that denatured with SDS.

\section{Haemagglutination and haemagglutination- inhibition tests}

The minimum concentration of type $\mathrm{A} \mathrm{HA}^{+}$-PTX to give haemagglutination was determined using neuraminidase-treated and non-treated (native) human erythrocytes. Non-treated erythrocytes were agglutinated by $0.098 \mu \mathrm{g}$ type A $\mathrm{HA}^{+}-\mathrm{PTX} \mathrm{ml}^{-1}$. Using the erythrocytes treated with neuraminidase $\left(\right.$ at $37^{\circ} \mathrm{C}$ for $1 \mathrm{~h}), 0 \cdot 195 \mu \mathrm{g}$ type $\mathrm{A} \mathrm{HA}^{+}-\mathrm{PTX} \mathrm{ml}^{-1}$ were needed to cause haemagglutination. Previously, Balding et al. (1973) reported that the HA activity of type A HA was activated by the treatment of erythrocytes with neuraminidase. This discrepancy might be ascribed to differences in the concentration of neuraminidase used, difference of neuraminidase and/or difference in the strains.

To elucidate the carbohydrate binding specificity of type A HA+-PTX, haemagglutination-inhibition tests were performed. Previously, Dasgupta \& Sugiyama (1977) reported that D-galactose and some of its derivatives were inhibitors of type A HA. Balding et al. (1973) proposed that type A HA is inhibited by D-galactose. In this study, seven kinds of saccharide were subjected to 
(a)

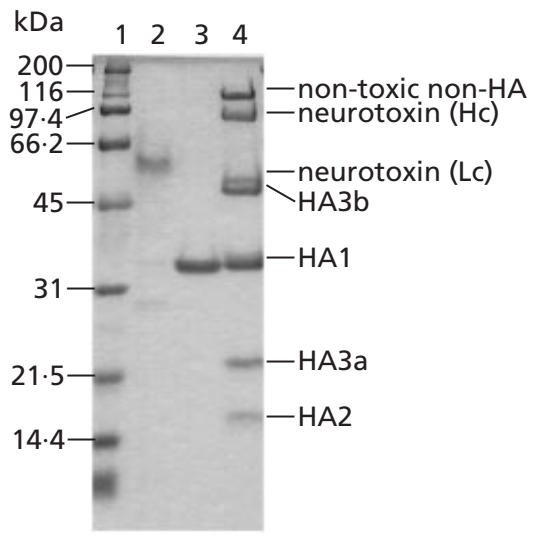

(b)

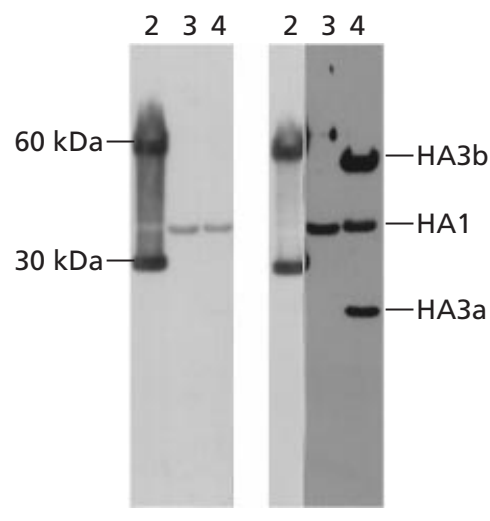

Fig. 2. SDS-PAGE (a) and immunoblots $(b, c)$ of purified type $\mathrm{HA}^{+}-\mathrm{PTX}$ and HA1. The samples were mixed with sample buffer containing $\beta$-mercaptoethanol and incubated at room temperature (lane 2 ) or heated at $100^{\circ} \mathrm{C}$ for 7 min (lanes 1, 3 and 4). Electrophoresis was performed on a $12.5 \%$ acrylamide gel. The gel was stained with CBB R-250 (a). Samples were blotted onto a PVDF membrane and successively reacted with the anti-type A HA1 serum (b) and anti-type A HA serum (c). Lane 1, protein standards; lanes 2 and 3, type A HA1; lane 4, type A HA+-PTX. (a)

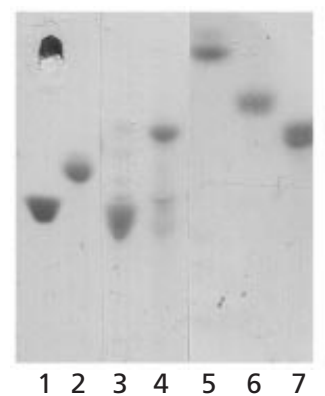

(b)

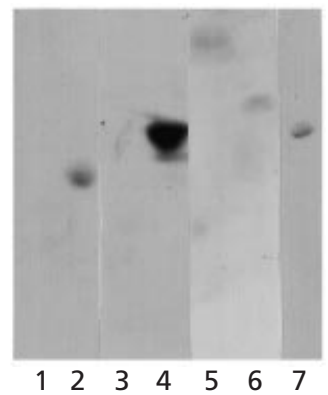

Fig. 3. Binding of type $\mathrm{AHA}^{+}-\mathrm{PTX}$ to glycolipids on TLC plates. TLC plates were developed in a solvent system with chloroform/methanol/water $(65: 35: 8$, by vol.). The plates were incubated with type A $\mathrm{HA}^{+}-\mathrm{PTX}$, stained by TLC-immunostaining as described in Methods (b) and then glycolipids were visualized by orcinol/ $\mathrm{H}_{2} \mathrm{SO}_{4}$ reagent (a). Glycolipids used are as follows: lane 1, GM1; lane 2, asialoGM1; lane 3, SPG; lane 4, paragloboside; lane 5, LacCer; lane 6, Gb3Cer; lane 7, Gb4Cer.

the inhibition tests. The HA activity of type $\mathrm{A} \mathrm{HA}^{+}$-PTX was inhibited by lactose, D-galactose, $\mathrm{N}$-acetyl-Dgalactosamine and D-fucose. To inhibit $50 \%$ of the HA activity $27 \cdot 5 \mathrm{mM}$ D-fucose, $15 \mathrm{mM} \mathrm{N}$-acetyl-D-galactosamine, $15 \mathrm{mM}$ D-galactose and $5 \mathrm{mM}$ lactose were required, whereas no inhibition was observed with treatment by $100 \mathrm{mM}$ D-glucose, $\mathrm{N}$-acetyl-D-glucosamine and D-mannose (data not shown).

\section{Binding of type A HA ${ }^{+}$-PTX to glycolipids}

The results from the haemagglutination tests and corresponding inhibition tests suggest that polysaccharides on the surface of human erythrocytes play an important role in the binding of type $\mathrm{A} \mathrm{HA}^{+}$-PTX. To explore this hypothesis further, we analysed the direct binding of type $\mathrm{A} \mathrm{HA}^{+}$-PTX to glycolipids by TLC-immunostaining.

As shown in Fig. 3, type A HA+ $\mathrm{HTX}^{+}$strongly bound to

Table 1. Binding of type A HA ${ }^{+}-$PTX to glycolipids

\begin{tabular}{|c|c|c|}
\hline Glycolipid & Structure & Binding \\
\hline GalCer & Gal $\beta 1$-Cer & - \\
\hline GlcCer & Glc $\beta 1$-Cer & - \\
\hline LacCer & Gal $\beta 1-4 \mathrm{Glc} \beta 1-\mathrm{Cer}$ & + \\
\hline Gb3Cer & Gal $\alpha 1-4 \mathrm{Gal} \beta 1-4 \mathrm{Glc} \beta 1-\mathrm{Cer}$ & + \\
\hline Gb4Cer & $\begin{array}{l}\text { GalNAc } \beta 1-3 \mathrm{Gal} \alpha 1-4 \mathrm{Gal} \beta \\
\text { 1-4Glc } \beta 1-\mathrm{Cer}\end{array}$ & + \\
\hline AsialoGM1 & $\begin{array}{l}\mathrm{Gal} \beta 1-3 \mathrm{GalNAc} \beta 1-4 \mathrm{Gal} \beta \\
1-4 \mathrm{Glc} \beta 1-\mathrm{Cer}\end{array}$ & ++ \\
\hline Paragloboside & $\begin{array}{l}\text { Gal } \beta 1-4 \mathrm{GlcNAc} \beta 1-3 \mathrm{Gal} \beta \\
1-4 \mathrm{Glc} \beta 1-\mathrm{Cer}\end{array}$ & +++ \\
\hline $\begin{array}{l}\text { Gangliosides } \\
\text { (GM1, GM2, } \\
\text { GM3, GM4, } \\
\text { GD1a, GD1b, } \\
\text { GD3, SPG) }\end{array}$ & & - \\
\hline $\begin{array}{l}\text { Sulfated } \\
\text { glycolipids (SM3, } \\
\text { SM4) }\end{array}$ & & - \\
\hline
\end{tabular}

paragloboside and asialoGM1 (Fig. 3b, lanes 2 and 4). Only weak binding was observed to Gb3Cer, Gb4Cer and LacCer (Fig. 3b, lanes 5-7). No binding was observed to GM1 and SPG (Fig. 3b, lanes 1 and 3). The results of direct binding tests of $\mathrm{HA}^{+}$-PTX to purified glycolipids are summarized in Table 1 . Type $\mathrm{A} \mathrm{HA}^{+}$PTX scarcely bond to gangliosides, GalCer, GlcCer or to sulfated glycosphingolipids (SM3, SM4). These results suggest that $\mathrm{N}$-acetylneuraminic acid or sulfate at the terminus of the carbohydrate structure disturbed the binding of type A $\mathrm{HA}^{+}$-PTX to glycolipids.

\section{Binding of type A HA+-PTX to glycoproteins}

To investigate the binding property of type A $\mathrm{HA}^{+}-\mathrm{PTX}$ to oligosaccharides, a binding test to enzyme-treated and non-treated glycoproteins was performed. Although 
(a)

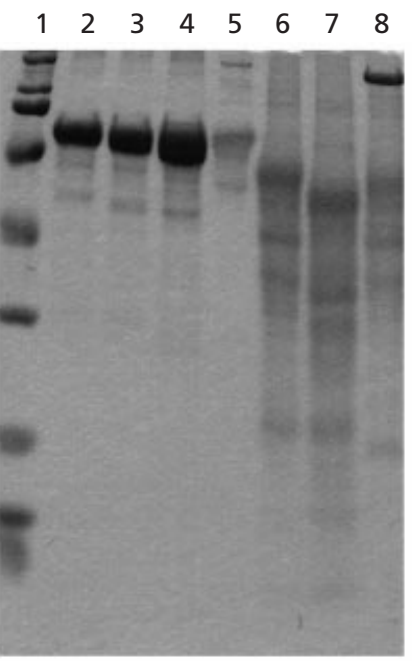

(b)

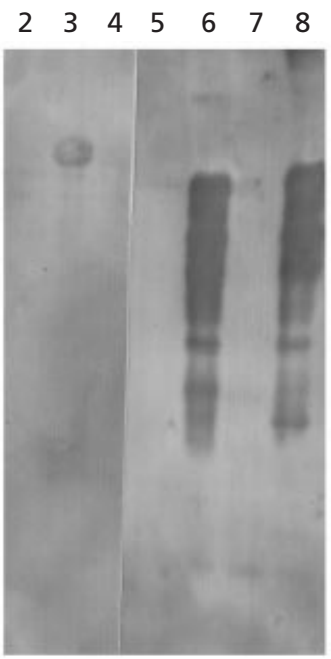

(c)

(d)

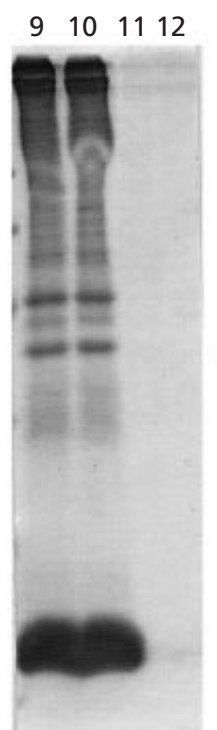

$9 \quad 101112$

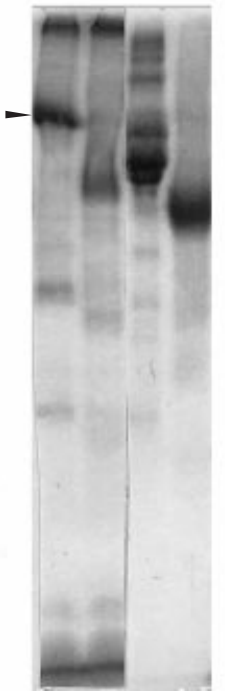

(e)

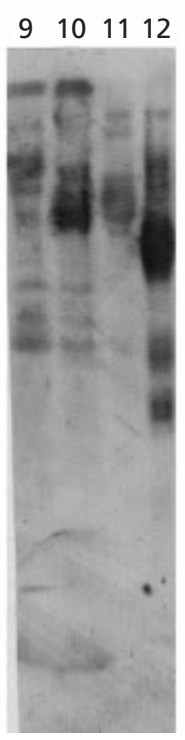

Fig. 4. Binding of type $A \mathrm{HA}^{+}-\mathrm{PTX}$ to glycoproteins and membrane proteins from human erythrocytes. Glycoproteins (10 $\mu \mathrm{g}$ each) and membrane proteins from human erythrocytes (300 $\mu \mathrm{g}$ each) were electrophoresed and stained with CBB R-250 $(a, c)$ or PAS (d) or electroblotted onto PVDF membranes for toxin binding analysis as described in Methods (b, e). Lane 1 contains protein standards. Glycoproteins used are as follows: lane 2, transferrin; lane 3, neuraminidase-treated transferrin; lane 4, glycopeptidase F-treated transferrin; lane 5, fetuin; lane 6, asialofetuin; lane 7, glycopeptidase F-treated asialofetuin; lane 8 , endo- $\alpha-N$-acetylgalactosaminidase-treated asialofetuin; lane 9, membrane proteins from human erythrocytes; lane 10, membrane proteins from neuraminidasetreated human erythrocytes; lane 11, human glycophorin $A$; lane 12, neuraminidase-treated glycophorin A.

type $\mathrm{A} \mathrm{HA}^{+}$-PTX did not bind to transferrin or fetuin (Fig. 4b, lanes 2 and 5), it bound to neuraminidasetreated transferrin and asialofetuin (Fig. 4b, lanes 3 and

6). As shown in Fig. 5a, transferrin contains two Nlinked di-branched sugar chains which are fully sialylated (complex type; Yamashita et al., 1993). Fetuin contains six carbohydrate moieties/molecules, at least three O-linked oligosaccharides and three $N$-linked oligosaccharides (Carr et al., 1993). The N-linked oligosaccharides are di- or tri-branched (complex type) and about $80 \%$ of these oligosaccharides are fully sialylated (Green et al., 1988). Therefore, our results indicate that type $\mathrm{A} \mathrm{HA}^{+}$-PTX bound to unsialylated oligosaccharides. $\mathrm{HA}^{+}$-PTX also bound to endo- $\alpha-N-$ acetylgalactosaminidase-treated asialofetuin (Fig. 4b, lane 8) but no binding was observed to glycopeptidase Ftreated transferrin and asialofetuin (Fig. 4b, lanes 4 and 7). Thus type A $\mathrm{HA}^{+}$-PTX binds to unsialylated $\mathrm{N}$ linked sugar chains (complex type) and not to three unsialylated O-linked sugar chains contained in fetuin. Since paragloboside and unsialylated $N$-linked sugar chains (complex type) which were bound by the toxin have a common structure, containing the Gal $\beta 1$ 4GlcNAc at the terminus of their oligosaccharides (Table 1, Fig. 5b), type A HA ${ }^{+}$-PTX should detect and bind to this structure of oligosaccharide. The Gal $\beta 1$ 3 GalNAc structure contained in asialoGM1 is also found in the desialylated O-linked sugar chain of glycoproteins. AsialoGM1 was detected by type $\mathrm{A} \mathrm{HA}^{+}-$ PTX, but desialylated O-linked sugar chains of fetuin were not. The structure of the sugar chain of asialoGM1 is Gal $11-3 \mathrm{GalNAc1-4Gal1-4Glc,}$ but that of desialylated O-linked sugar chain is only Gal $\beta 1-3 \mathrm{GalNAc}$. Thus, not only Gal $\beta 1-3 \mathrm{GalNAc}$ but also the whole sugar chain of asialoGM1 may be important for the binding of toxin. Alternatively, O-linked sugar chains of fetuin may not be exposed to the surface of the molecule, unlike asialoGM1.

\section{Binding of type A HA+-PTX to glycoproteins of erythrocytes and binding of erythrocytes to type $A$ HA+-PTX}

To confirm which glycoproteins of erythrocytes were detected by type A HA ${ }^{+}$-PTX, membrane proteins were prepared from erythrocytes and the binding of toxin was analysed. The membrane proteins prepared from non-treated and neuraminidase-treated erythrocytes exhibited multiple protein bands on SDS-PAGE by CBB staining (Fig. 4c, lanes 9 and 10). The results show that mainly a single band was bound by type $\mathrm{A} \mathrm{HA}^{+}$-PTX in each preparation (Fig. 4e, lanes 9 and 10). The mobilities of these bands were identical to those detected by periodic acid-Schiff (PAS) staining (Fig. 4d). The PASpositive band indicated by the arrow in Fig. $4 \mathrm{~d}$ is considered to be a dimeric form of glycophorin A (PAS1) from a previous report (Furthmayr et al., 1975). Commercial glycophorin A and neuraminidase-treated glycophorin A were also bound by type A HA+ $\mathrm{H}^{+} \mathrm{PT}$ (Fig. 4e, lanes 11 and 12). These protein bands were not detected by $\mathrm{CBB}$ staining, but the mobilities of protein bands bound by type A $\mathrm{HA}^{+}$-PTX were identical to those detected by PAS staining. In the other experiment, the binding of erythrocytes to type A HA+-PTX-coated 96- 
(a)
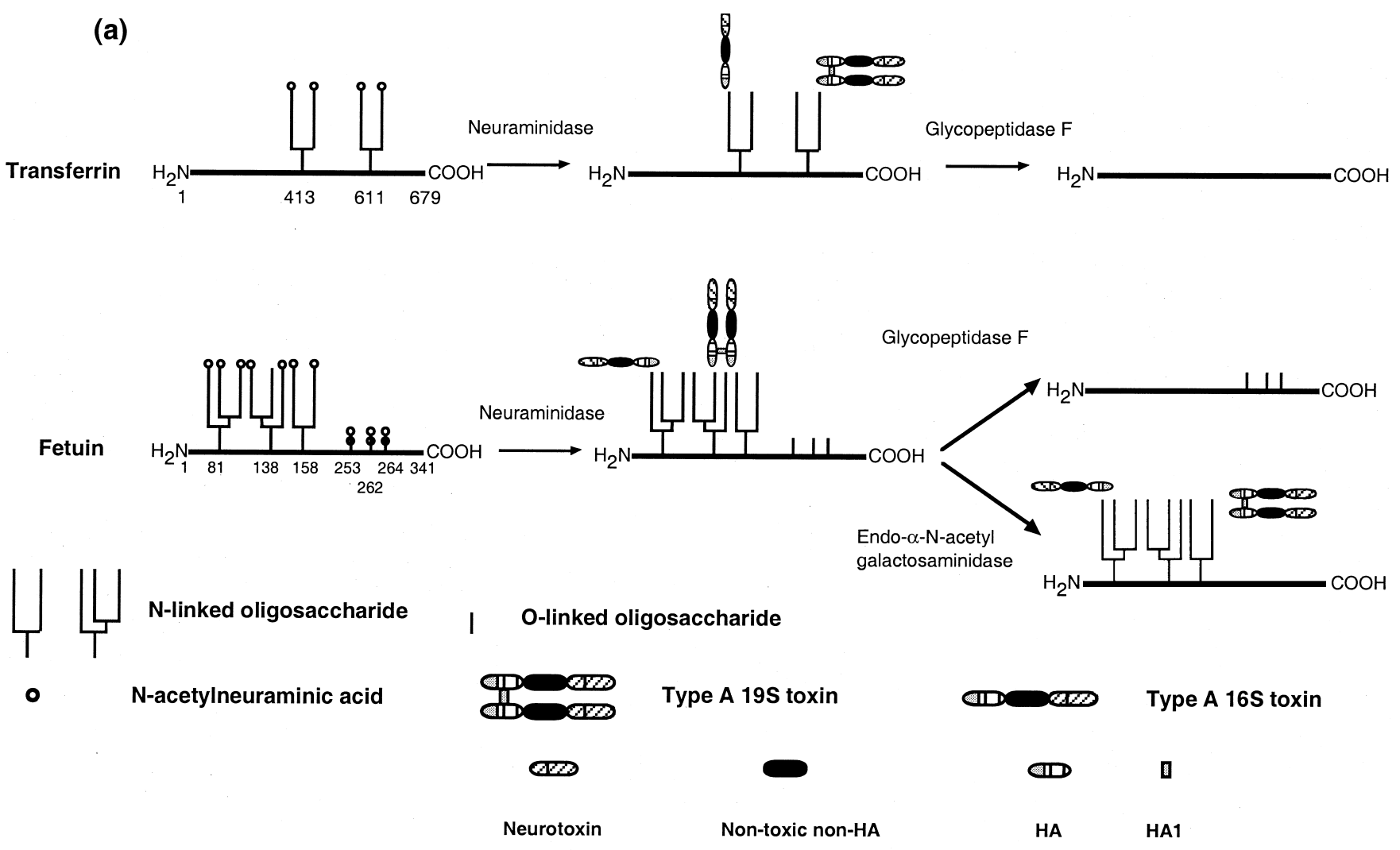

(b)

$\mathrm{N}$-linked oligosaccharide (complex type)
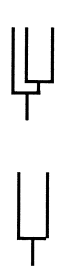

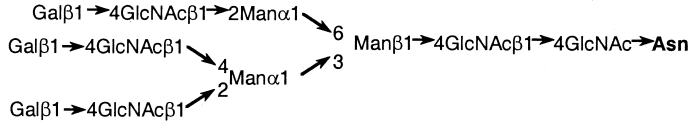

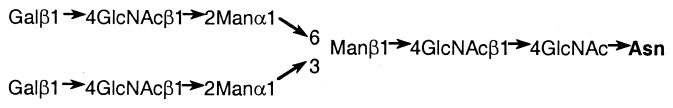

Gal $\beta 1 \rightarrow 3$ GalNAc $\rightarrow$ Ser or Thr

O-linked oligosaccharide

I

NeuAc $\alpha$

Fig. 5. The proposed structures of native and enzyme-treated glycoproteins and binding of type A HA ${ }^{+}-$PTX (a) and the typical structures of $N$-linked (complex type) and $O$-linked sugar chains (b). The structures of HA ${ }^{+}-$PTX (19S and 16S toxins) are cited from our previous report (Oguma et al., 1999).

well microtitre plates was analysed (Fig. 6a, b). Nontreated and neuraminidase-treated erythrocytes both bound to type $\mathrm{A} \mathrm{HA}^{+}$-PTX, and the binding was not affected by the presence of fetuin. Contrary to this, dosedependent inhibition was observed by the addition of asialofetuin, glycophorin A and neuraminidase-treated glycophorin A to the reaction mixture. However, the concentration of glycoproteins required for inhibition of neuraminidase-treated erythrocyte binding seemed to be higher than that required for non-treated erythrocyte binding inhibition.

Glycophorin A is a major glycoprotein of the membrane of human erythrocytes (Marchesi et al., 1972) and the molecule contains approximately 15 O-linked sugar chains and one $\mathrm{N}$-linked sugar chain (Tomita et al., 


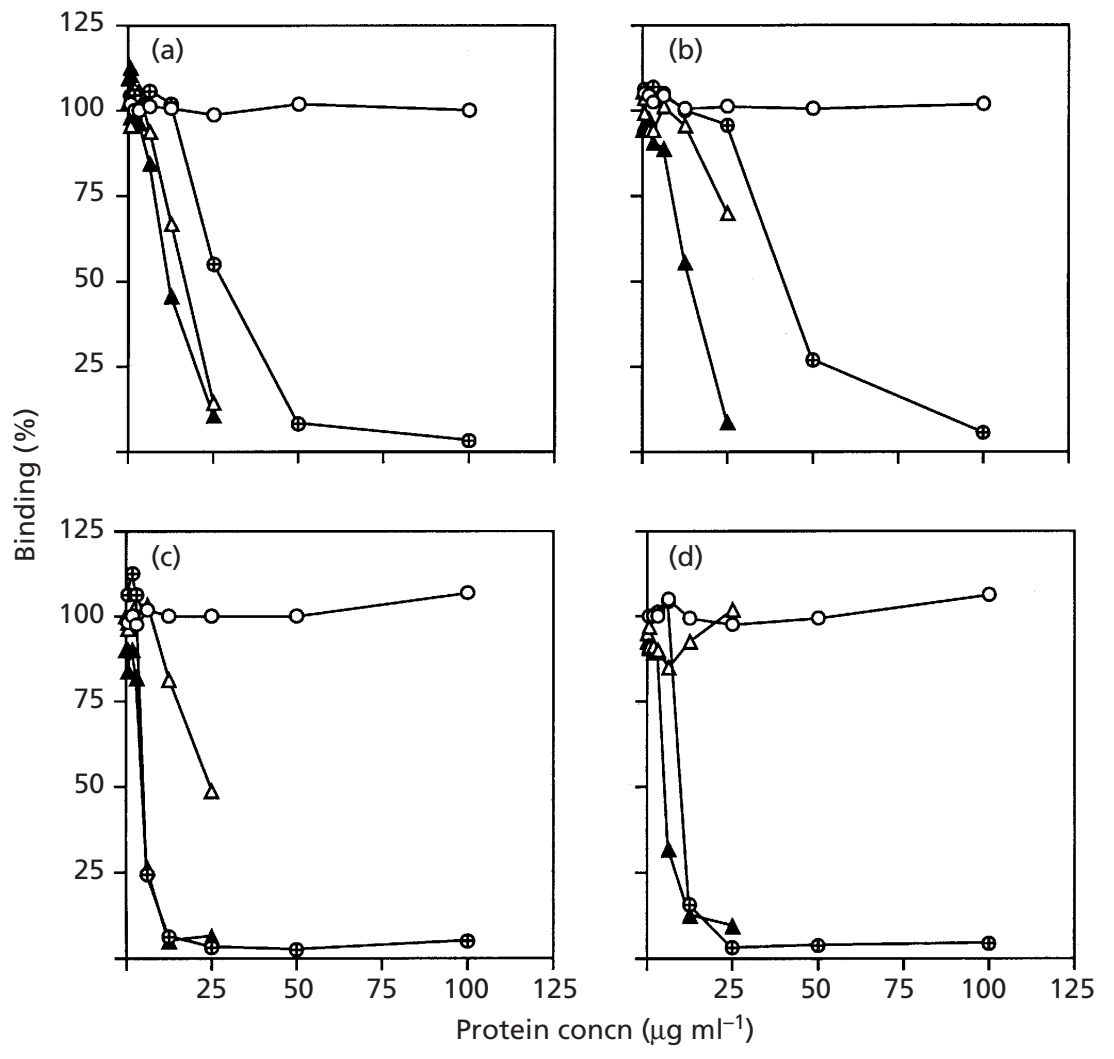

Fig. 6. Binding of erythrocytes to type $A$ $\mathrm{HA}^{+}$-PTX and HA1 on microtitre plates. Type A HA-PTX $(a, b)$ and HA1 (c, d) were coated on plates, and incubated in the presence of fetuin $(\bigcirc)$, asialofetuin $(\oplus)$, glycophorin $A$ $(\triangle)$ or asialoglycophorin $A(\boldsymbol{\Delta})$. Native erythrocytes $(a, c)$ or neuraminidase-treated erythrocytes $(b, d)$ bound to type A HA H $^{+}$PTX or HA1 were lysed and the amount of bound erythrocytes was determined spectrophotometrically at $405 \mathrm{~nm}$ as described in Methods. The amount of bound erythrocytes in the absence of glycoprotein was determined as $100 \%$.
1975). The structure of the $\mathrm{N}$-linked oligosaccharide is a dibranched complex type sugar chain having $\mathrm{N}$ acetylglucosamine linked at the mannosyl residue of the core portion and fucose linked at the proximal $\mathrm{N}$ acetylglucosamine residue. More than $50 \%$ of this sugar chain is monosialylated at the C-6 position of one of two terminal galactoses, and the other terminal galactose is not sialylated. The remaining $\mathrm{N}$-linked sugar chains are disialylated (Yoshida et al., 1980). Therefore, $\mathrm{HA}^{+}$PTX can agglutinate erythrocytes by binding to one unsialylated branch of an $N$-linked sugar chain in glycophorin A in the case of non-treated erythrocytes and by binding to two desialylated branches of the protein in the case of neuraminidase-treated erythrocytes.

\section{Characterization of HA1}

Haemagglutination tests, and binding tests to glycolipids, glycoproteins and erythrocytes were also performed using purified type A HA1. Purified HA1 did not agglutinate native erythrocytes even though $400 \mu \mathrm{g} \mathrm{ml}^{-1}$ was used. However, neuraminidase-treated erythrocytes were agglutinated by $100 \mu \mathrm{g}$ HA $1 \mathrm{ml}^{-1}$. Type A HA1 bound to paragloboside and asialoGM1 similar to $\mathrm{HA}^{+}-$ PTX. However, binding to the other glycolipids was not observed (Fig. 7b). HA1 did not bind to native transferrin and fetuin, but bound to neuraminidase-treated transferrin and asialofetuin (Fig. 7c). HA1 also bound to endo- $\alpha-N$-acetylgalactosaminidase-treated asialofetuin, but did not bind to glycopeptidase F-treated transferrin and asialofetuin (Fig. 7c) as observed in $\mathrm{HA}^{+}-\mathrm{PTX}$. Native erythrocytes bound to purified type A HA1 the same as $\mathrm{HA}^{+}$-PTX and the binding was not affected by fetuin. The binding of erythrocytes to HA1 was reduced by asialofetuin, glycophorin A and neuraminidasetreated glycophorin A (Fig. 6c). The effect of glycophorin A seemed to be smaller than that of asialofetuin and neuraminidase-treated glycophorin A. Using erythrocytes treated with neuraminidase, inhibition by glycophorin A was not observed (Fig. 6d). The similar binding specificities of $\mathrm{HA} 1$ and $\mathrm{HA}^{+}$-PTX indicates that type A $\mathrm{HA}^{+}$-PTX binds to glycolipids, glycoproteins and erythrocytes through HA1. We previously reported that $\mathrm{HA}^{+}$-PTX and HA1 bind via galactose moieties using recombinant HA subcomponents (GST-fusion proteins) expressed in Escherichia coli (Fujinaga et al., 2000). Fu et al. (1998) also reported that polyclonal antibodies against HA1 inhibit the haemagglutination caused by $\mathrm{HA}^{+}-\mathrm{PTX}$ and that the toxin agglutinates erythrocytes through HA1. These results support the conclusions obtained in this study.

Orally ingested botulinum progenitor toxins are supposed to be absorbed from the upper intestine into the lymphatic system (Sugii et al., 1977). Recently, we proposed that type C HA plays an important role in the absorption of the progenitor toxins from the small intestine of guinea pigs (Fujinaga et al., 1997). We also reported that type $\mathrm{A} \mathrm{HA}^{+}$-PTX and GST-HA1 bound to epithelial cells of the guinea pig small intestine and that their binding was reduced by galactose and lactose 
(a)

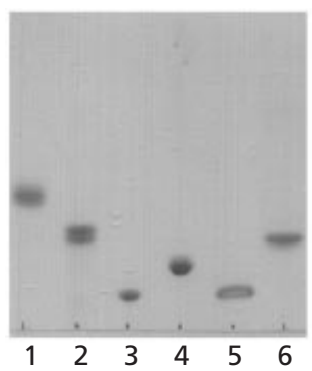

(b)

(c)

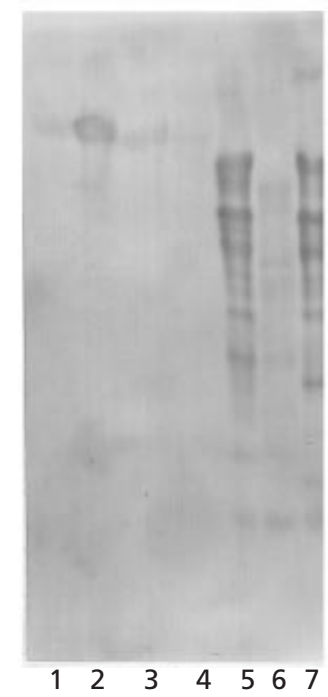

$\begin{array}{lllllll}1 & 2 & 3 & 4 & 5 & 6 & 7\end{array}$

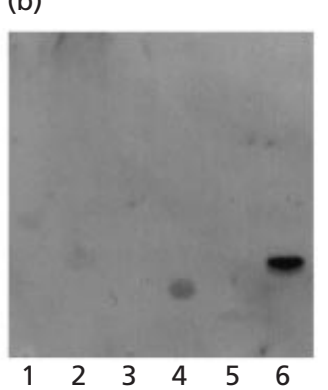

$\begin{array}{llllll}1 & 2 & 3 & 4 & 5 & 6\end{array}$

Fig. 7. Binding of HA1 to glycolipids and glycoproteins. The TLC plates were developed in a solvent system with chloroform/methanol/water (65:35:8, by vol.) The plates were incubated with type A HA1, stained by TLC-immunostaining as described in Methods (b) and then glycolipids were visualized by orcinol $/ \mathrm{H}_{2} \mathrm{SO}_{4}$ reagent (a). Glycolipids used are as follows: lane 1, Gb3Cer; lane 2, Gb4Cer; lane 3, GM1; lane 4, asialoGM1; lane 5, SPG; lane 6, paragloboside. (c) Glycoproteins were electrophoresed, electroblotted onto a PVDF membrane and binding of HA1 was analysed. Glycoproteins (10 $\mu \mathrm{g}$ each) used are as follows: lane 1, transferrin; lane 2, neuraminidasetreated transferrin; lane 3, glycopeptidase F-treated transferrin; lane 4, fetuin; lane 5, asialofetuin; lane 6, glycopeptidase F-treated asialofetuin; lane 7 , endo- $\alpha-N$ acetylgalactosaminidase-treated asialofetuin.

(Fujinaga et al., 2000). While Maksymowych \& Simpson (1998) found that type A neurotoxin itself might be absorbed, by performing in vitro experiments with human carcinoma cells, they also reported that the neurotoxin as well as $\mathrm{HA}^{+}$-PTX was absorbed from the stomach and small intestine using an in vivo mouse model (Maksymowych et al., 1999). In this study, we have demonstrated that the type $\mathrm{A} \mathrm{HA}^{+}$-PTX binds to some glycolipids and glycoproteins, but does not bind to gangliosides. However, it has been reported previously that type A neurotoxin binds to gangliosides but does not bind to some neutral glycolipids (Kamata et al., 1997). Therefore, it is postulated that unsialylated oligosaccharides on the surface of the small intestine are

the receptors at least for type A $\mathrm{HA}^{+}$-PTX (not neurotoxin). Neurotoxin and/or $12 \mathrm{~S}$ toxin may have a different receptor and/or different adsorptive pathway from those of $\mathrm{HA}^{+}$-PTX. The mechanisms of how HApositive and -negative toxins (including neurotoxin alone) are absorbed and enter the lymphatic vessel through the epithelial cells is a topic for further analysis.

\section{ACKNOWLEDGEMENTS}

We thank Professor Shinsei Gasa (Sapporo Medical University) for preparation of the SPG used.

This work was supported by a grant 10770118 from the Ministry of Education, Science and Culture of Japan, and by a grant for the 'Emerging and Re-emerging Infectious Disease' funded by the Ministry of Health and Welfare of Japan.

\section{REFERENCES}

Balding, P., Gold, E. R., Boroff, D. A. \& Roberts, T. A. (1973). Observations on receptor specific proteins. II. Hemagglutination and hemagglutination-inhibition reactions of Clostridium botulinum types A, C, D, and E hemagglutinins. Immunology 25, 773-782.

Carr, S. A., Huddleston, M. J. \& Bean, M. F. (1993). Selective identification and differentiation of $\mathrm{N}$ - and O-linked oligosaccharides in glycoproteins by liquid chromatography-mass spectrometry. Protein Sci 2, 183-196.

Dasgupta, B. R. \& Sugiyama, H. (1977). Inhibition of Clostridium botulinum type A and B hemagglutinins by sugars. Can J Microbiol 23, 1257-1260.

Fu, F. N., Sharma, S. K. \& Singh, B. R. (1998). A protease-resistant novel hemagglutinin purified from type A Clostridium botulinum. J Protein Chem 17, 53-60.

Fujinaga, Y., Inoue, K., Shimazaki, S. \& 8 other authors (1994). Molecular construction of Clostridium botulinum type C progenitor toxin and its gene organization. Biochem Biophys Res Commun 205, 1291-1298.

Fujinaga, Y., Inoue, K., Watanabe, S., Yokota, K., Hirai, Y., Nagamachi, E. \& Oguma, K. (1997). The haemagglutinin of Clostridium botulinum type $\mathrm{C}$ progenitor toxin plays an essential role in binding of toxin to the epithelial cells of guinea pig small intestine, leading to the efficient absorption of the toxin. Microbiology 143, 3841-3847.

Fujinaga, Y., Inoue, K., Nomura, T., Sasaki, J., Marvayd, J. C., Popoff, M. R., Kozaki, S. \& Oguma, K. (2000). Identification and characterization of functional subunits of Clostridium botulinum type A progenitor toxin involved in binding to intestinal microvilli and erythrocytes. FEBS Lett 467, 179-183.

Furthmayr, H., Tomita, M. \& Marchesi, V. T. (1975). Fractionation of the major sialoglycopeptides of the human red blood cell membrane. Biochem Biophys Res Commun 65, 113-121.

Gasa, S., Makita, A. \& Kinoshita, Y. (1983). Further study of the chemical structure of the equine erythrocyte hematoside containing O-acetyl ester. J Biol Chem 258, 876-881.

Green, E. D., Adelt, G., Baenzinger, J. U., Wilson, S. \& Halbeek, H. V. (1988). The asparagine-linked oligosaccharides on bovine fetuin. J Biol Chem 263, 18253-18268.

Hirano, H. \& Watanabe, T. (1990). Microsequencing of proteins electrotransferred onto immobilizing matrices from polyacrylamide gel electrophoresis: application to an insoluble protein. Electrophoresis 11, 573-580. 
Hoschutzky, I., Lottspeich, F. \& Jann, K. (1989). Isolation and characterization of the alpha-galactosyl-1,4-beta-galactosylspecific adhesin ( $\mathrm{P}$ adhesin) from fimbriated Escherichia coli. Infect Immun 57, 76-81.

Inoue, K., Fujinaga, Y., Watanabe, T., Ohyama, T., Takeshi, K., Moriishi, K., Nakajima, H., Inoue, K. \& Oguma, K. (1996). Molecular composition of Clostridium botulinum type A progenitor toxins. Infect Immun 64, 1589-1594.

Inoue, K., Fujinaga, Y., Honke, K. \& 7 other authors (1999). Characterization of haemagglutinin activity of Clostridium botulinum type C and D $16 \mathrm{~S}$ toxins, and one subcomponent of haemagglutinin (HA1). Microbiology 145, 2533-2542.

Kamata, Y., Yoshimoto, M. \& Kozaki, S. (1997). Interaction between botulinum neurotoxin type A and ganglioside: ganglioside inactivates the neurotoxin and quenches its tryptophan fluorescence. Toxicon 35, 1337-1340.

Laemmli, U. K. (1970). Cleavage of structural proteins during the assembly of the head of bacteriophage T4. Nature 227, 680-685.

Maksymowych, A. B. \& Simpson, L. L. (1998). Binding and transcytosis of botulinum neurotoxin by polarized human colon carcinoma cells. J Biol Chem 273, 21950-21957.

Maksymowych, A. B., Reinhard, M., Malizio, C. J., Goodnough, M. C., Johnson, E. A. \& Simpson, L. L. (1999). Pure botulinum neurotoxin is absorbed from the stomach and small intestine and produces peripheral neuromuscular blockade. Infect Immun 67, 4708-4712.

Marchesi, V. T., Tillack, T. W., Jackson, R. L., Segrest, J. P. \& Scott, R. E. (1972). Chemical characterization and surface orientation of the major glycoprotein of the human erythrocyte membrane. Proc Natl Acad Sci US A 69, 1445-1449.

Oguma, K., Inoue, K., Fujinaga, Y., Yokota, K., Watanabe, T., Ohyama, T., Takeshi, K. \& Inoue, K. (1999). Structure and function of Clostridium botulinum progenitor toxin. J Toxicol-Toxin Rev $18,17-34$.

Ohishi, I. \& Sakaguchi, G. (1980). Oral toxicities of Clostridium botulinum type $\mathrm{C}$ and $\mathrm{D}$ toxins of different molecular sizes. Infect Immun 28, 303-309.

Ohishi, I., Sugii, S. \& Sakaguchi, G. (1977). Oral toxicities of Clostridium botulinum toxins in response to molecular size. Infect Immun 16, 107-109.

Sakaguchi, G., Kozaki, S. \& Ohishi, I. (1984). Structure and function of botulinum toxins. In Bacterial Protein Toxins, pp. 435-443. Edited by J. E. Alouf, F. J. Fehrenbach, J. H. Freer \& J. Jeljasawicz. London: Academic Press.

Sugii, S., Ohishi, I. \& Sakaguchi, G. (1977). Correlation between oral toxicity and in vitro stability of Clostridium botulinum type $\mathrm{A}$ and B toxins of different molecular sizes. Infect Immun 16, 910-914.

Tomita, M., Furthmayr, H. \& Marchesi, V. T. (1975). Primary structure of human erythrocyte glycophorin A: isolation and characterization of peptides and complete amino acid sequence. Biochemistry 17, 4756-4770.

Yamashita, K., Ideo, H., Ohkura, T., Fukushima, K., Yuasa, I., Ohno, K. \& Takeshita, K. (1993). Sugar chains of serum transferrin from patients with carbohydrate deficient glycoprotein syndrome: evidence of asparagine- $\mathrm{N}$-linked oligosaccharide transfer deficiency. J Biol Chem 268, 5783-5789.

Yoshida, H., Furthmayr, H. \& Kobata, A. (1980). Structure of the asparagine-linked sugar chains of glycophorin A. J Biol Chem 255, 9713-9718.

Received 25 July 2000; revised 20 December 2000; accepted 10 January 2001. 